\title{
Radiation Driven Atmospheres of O-type stars: Constraints on the Mass-Luminosity Relation of Central Stars of Planetary Nebulae
}

\author{
A.W.A. Pauldrach(1), T.L. Hoffmann(1), R.H. Méndez(1,2) \\ (1) Institut für Astronomie und Astrophysik der Universität München, \\ Scheinerstraße 1, 81679 München, Germany; \\ (2) Institute for Astronomy, University of Hawaii, \\ 2680 Woodlawn Drive, Honolulu, HI 96822, U.S.A.
}

\begin{abstract}
Recent advances in the modelling of stellar winds driven by radiation pressure make it possible to fit many wind-sensitive features in the UV spectra of hot stars, opening the way for a hydrodynamically consistent determination of stellar radii, masses, and luminosities from the UV spectrum alone. It is thus no longer necessary to assume a theoretical mass-luminosity relation. As the method has been shown to work for massive $\mathrm{O}$ stars, we are now able to test predictions from the postAGB evolutionary calculations quantitatively for the first time. Here we present the first rather surprising consequences of using the new generation of model atmospheres for the analysis of a sample of central stars of planetary nebulae.
\end{abstract}

\section{Introduction}

A lot of work on model atmospheres of PN central stars (CSPNs in what follows) has been motivated by the desire to obtain information about the basic properties of CSPNs (surface temperature, mass, luminosity, abundances), so as to be able to test predictions from post-AGB evolutionary calculations. The earlier efforts, based on plane-parallel non-LTE models, could not achieve a completely independent test, in the following sense: since the plane-parallel model fits to $\mathrm{H}$ and $\mathrm{He}$ photospheric absorption lines can only produce information about surface temperature, He abundance and $\log g$, we cannot derive stellar masses or luminosities, but only $L / M$ ratios. This is exactly the same problem we face when dealing with low-gravity early-type "supergiant" stars at high Galactic latitudes: are they luminous and massive, or are they evolving away from the AGB? We need some independent evidence to settle the issue - for example, the distance to the star. Unfortunately, we lack reliable distances to most CSPNs.

So what could be done was to plot the positions of CSPNs in the $\log g$ $\log T_{\text {eff }}$ diagram, and compare them with plots of post-AGB tracks, translated from the $\log L-\log T_{\text {eff }}$ diagram. After doing this translation it is possible to read the stellar mass in the $\log g$ - $\log T_{\text {eff }}$ diagram. From this, we can derive $L$ and, if we know the visual dereddened apparent magnitude, a so-called "spectroscopic distance". All this work, however, is based on assuming that the evolutionary 
models give us the correct relation between stellar mass and luminosity. It is not a real test of the evolutionary models, but only a consistency check.

In the last 10 years there has been a lot of progress in the modelling of stellar winds driven by radiation pressure. Many CSPNs show spectroscopic evidence of winds, in the form of emission lines in the visible spectrum and especially P-Cygni-type profiles of resonance lines in the ultraviolet between 1000 and $2000 \AA$.

The existence of these wind features provides both a challenge and an opportunity. The challenge is to model them. The opportunity is to use the information about the geometrical extension of the atmosphere and the forces of gravity and radiative pressure, implicit in the wind profiles (from which the terminal velocity and mass loss rate can be derived), to obtain the physical size of the star, which is the key to derive the stellar luminosity and mass. (The idea is described together with a first application by Pauldrach et al. 1988.) Thus the successful modelling of the wind features opens the way for a real test of the mass-luminosity relation of CSPNs.

In this review we would like to present the current situation of the project and the rather surprising results obtained up to now. Section 2 describes a model analysis of a massive Population I star, using state-of-the-art hydrodynamically consistent, spherically symmetric model atmospheres to demonstrate the power of the technique and to show how successfully we can reproduce the ionizing fluxes and observed spectra of such stars. In Section 3 we introduce the windmomentum-luminosity relation and describe a previous attempt to determine whether or not CSPNs follow this relation. In Section 4 we show two examples of the application of our hydrodynamically consistent wind models to CSPNs (fits to IUE and HST spectra and derived parameters). In Section 5 we add the results from 6 other CSPNs similarly analyzed and discuss the consequences.

\section{Modelling a massive $\mathrm{O}$ supergiant: $\alpha \mathrm{Cam}$}

The analysis method is described in detail by Pauldrach et al. (2001). ${ }^{3}$ Here we can only give a brief summary. The analysis method is based on modelling a homogeneous, stationary, extended, outflowing, spherically symmetric radiation driven atmosphere. A complete model atmosphere calculation involves solving the hydrodynamics and the NLTE problem (rate equations, radiative transfer). The solution of the total interdependent system of equations is obtained iteratively. This permits the calculation of the predicted or synthetic spectrum, which is then compared to the observed UV spectrum. The process is repeated with different stellar parameters until a satisfactory fit is obtained.

The UV spectrum between 1000 and $2000 \AA$ carries a lot of information: P-Cygni-type profiles of resonance lines of several ions of $\mathrm{C}, \mathrm{N}, \mathrm{O}, \mathrm{Si}, \mathrm{S}, \mathrm{P}$, as well as hundreds of strongly wind-contaminated lines of Fe IV, Fe v, Fe vI, Cr v, Ni IV, ArV, ArVI. But the information about the stellar parameters can be extracted only after careful analysis. A very important recent improvement of

\footnotetext{
${ }^{3}$ This paper is also available on the Web, at http://www.usm.uni-muenchen.de/people/adi/adi.html.
} 


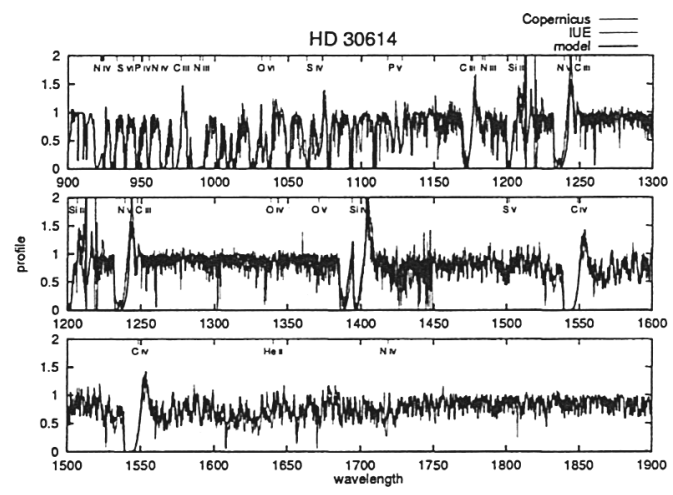

Figure 1. Synthetic UV spectrum of a model for $\alpha$ Cam compared to the observations by Copernicus and IUE, demonstrating the quality that can be achieved with our new model generation.

our method concerns the development of a substantially consistent treatment of the blocking and blanketing influence of all metal lines in the entire sub- and supersonically expanding atmosphere. All the results we will present are based on this new generation of models.

The operational procedure is as follows. A preliminary inspection of the visual or UV spectrum of the star to be analyzed gives an initial estimate of $T_{\text {eff }}$. From the UV spectrum, the terminal wind velocity $v_{\infty}$ can be measured directly. Now an initial value for the stellar radius $R$, defined at a Rosseland optical depth of $2 / 3$, is assumed. Using this $R$ and $v_{\infty}$ we obtain an estimate of the mass $\left(v_{\infty}\right.$ scales with $\sqrt{M / R}$ as explained by the theory of radiation driven winds). With the current values of $R, T_{\text {eff }}, M$, and assuming a set of abundances, we can solve the model atmosphere and calculate the velocity field, the mass loss rate $\dot{M}$, and the synthetic spectrum. Now this predicted spectrum is compared to the observed one. If the fit is not satisfactory, we need to modify $\dot{M}$ via a change of $R$ (since $\log \dot{M} \sim \log L$, according to the radiation driven wind theory). The change in $R$ forces us to change the mass, too, in order to keep $v_{\infty}$ consistent with the observed value. The new model is calculated and the process is repeated until we obtain a good fit to all features in the observed spectrum.

Figure 1 shows the result of applying this procedure to the $\mathrm{O}$ supergiant HD $30614(\alpha \mathrm{Cam})$. The final parameters are $T_{\text {eff }}=29000 \mathrm{~K}, \log g=3.0$, $R=30 R_{\odot}, \dot{M}=4 \times 10^{-6} M_{\odot} \mathrm{yr}^{-1}, v_{\infty}=1500 \mathrm{~km} / \mathrm{s}$. (Note that the consistent treatment of the hydrodynamics in our procedure will be described in a forthcoming paper (Pauldrach and Hoffmann 2002); the method is also illustrated in these proceedings by Hoffmann and Pauldrach.) This implies a stellar mass of $33 M_{\odot}$ and a spectroscopic distance of $1.2 \mathrm{kpc}$, in good agreement with the distance estimate of $1 \mathrm{kpc}$ (Scuderi et al. 1998). Thus we show that our current models produce satisfactory results for massive Population I stars. Can we apply the same procedure to CSPNs?

\section{The relation between wind-momentum loss rate and luminosity}

The radiatively driven wind theory predicts, for fixed abundances, a simple relation between the quantity $\dot{M} v_{\infty}$, which has the dimensions of a momentum 


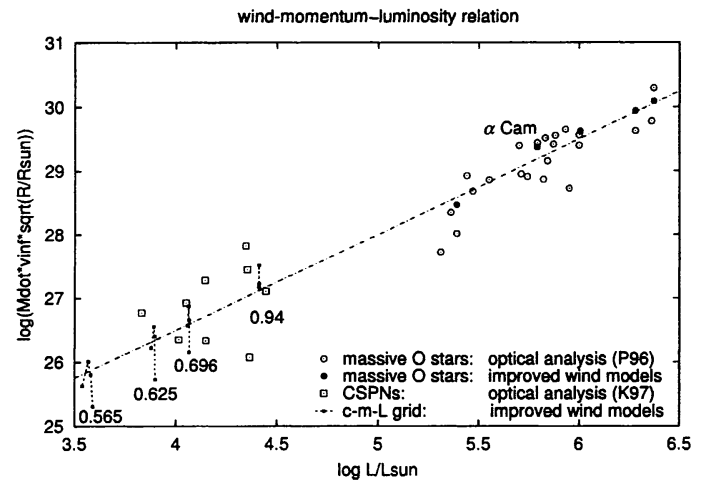

Figure 2. The wind-momentum-luminosity relation for massive $\mathrm{O}$ stars and CSPNs. P96 designates the analysis based on $\mathrm{H} \alpha$ profiles by Puls et al. 1996, K97 that of CSPNs by Kudritzki et al. 1997. Also plotted are the calculated wind momenta for a sample of massive $\mathrm{O}$ stars and for a grid of stars following post-AGB evolutionary tracks (masses given in $M_{\odot}$ ).

loss rate, and the stellar luminosity:

$$
\dot{M} v_{\infty} \sim R^{-0.5} L^{(1 / \alpha)}
$$

where $\alpha$, the power law exponent of the line strength distribution function, is $\simeq 2 / 3$ (slightly dependent on temperature and metallicity; see, for example, Puls et al. 1996). It is practical to plot the $\log$ of $\dot{M} v_{\infty} R^{0.5}$ as a function of $\log L$. In this kind of plot the theory predicts, in first approximation, a linear relation, which is indeed followed by all kinds of massive hot stars, as shown in Figure 2.

An initial attempt to verify if CSPNs follow the wind-momentum-luminosity relation was partly successful (see Figure 3 in Kudritzki et al. 1997 and also our Figure 2). In that paper $Q$-values (a quantity relating mass loss rate and stellar radius, $\left.Q \sim \dot{M}\left(R v_{\infty}\right)^{-3 / 2}\right)$ were derived from observed $\mathrm{H} \alpha$ profiles, and the stellar masses were derived from $T_{\text {eff }}$ and $\log g$, using post-AGB tracks plotted in the $\log g-\log T_{\text {eff }}$ diagram. The stellar radii (and thus, mass loss rates) and luminosities were then obtained from the masses and the post-AGB mass-luminosity relation. The CSPNs were found to be at the expected position along the windmomentum-luminosity relation, indicating a qualitatively successful prediction by the theory of radiatively driven winds. However, the situation was not satisfactory because there appeared to be a large dispersion in wind strengths at a given luminosity (strong-winded and weak-winded CSPNs) and some of the CSPN masses and luminosities were very high $\left(M>0.8 M_{\odot}\right)$, in contradiction with theoretical post-AGB evolutionary speeds

Thus, at that point we had a qualitative positive result, namely that in principle the CSPN winds obey the same physics as the massive $\mathrm{O}$ star winds; but we also had some unsolved problems which we now want to rediscuss using the improved model atmospheres.

As a first step we have used our models to calculate the terminal velocities and mass loss rates for a grid of stars following the current theoretical postAGB evolutionary tracks (see, for instance, Blöcker 1995); the resulting wind momenta are also plotted in Figure 2. The numerical models do nicely follow the expected theoretical wind-momentum-luminosity relation, although the spread is much smaller than that in the values derived by Kudritzki et al. Furthermore, the location of the observed sample in the diagram indicates masses between 0.6 and $0.95 M_{\odot}$, with a clear absence of CSPNs with masses below $0.6 M_{\odot}$. 


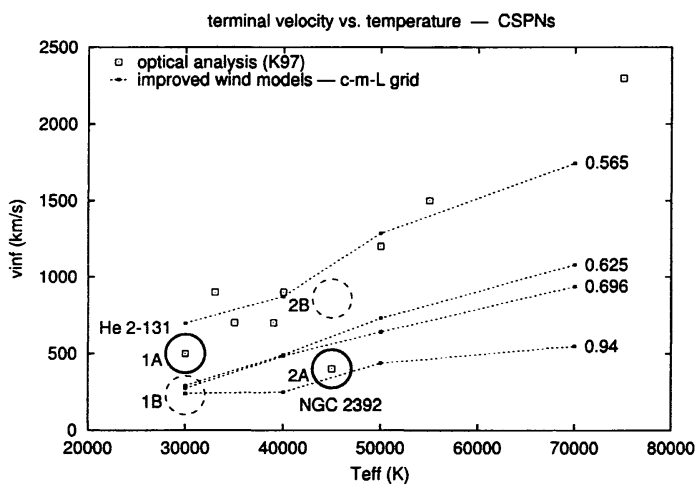

Figure 3. Terminal velocities calculated for a grid of stars following post-AGB evolutionary tracks (dashed lines, masses in $M_{\odot}$ labelled on the right) compared to observed values (squares).

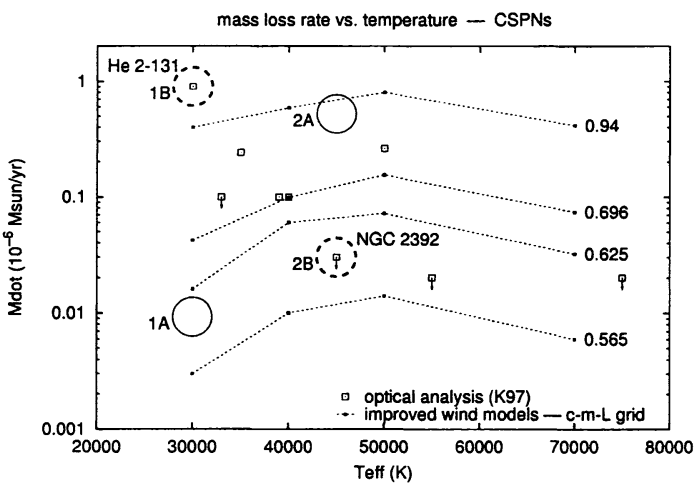

Figure 4. Mass loss rates computed for the above grid of stars, compared to the values derived by Kudritzki et al. (1997) for the same set of observations as in Figure 3.

This result is entirely unexpected from the standpoint of current evolutionary theory. To understand this discrepancy, we must compare the relations of the individual dynamic quantities, $v_{\infty}$ and $\dot{M}$. This is done in Figures 3 and 4: Figure 3 shows our calculated terminal velocities and the observed values, Figure 4 shows our computed mass loss rates and those derived by Kudritzki et al. for their sample.

Here, too, a fundamental discrepancy immediately becomes obvious: whereas the positions of the observations in the diagram showing the terminal velocities cluster at rather small CSPN masses (between 0.5 and $0.6 M_{\odot}$ ), their mass loss rates point to a majority of masses above $0.7 M_{\odot}$.

A detailed look at the positions of individual CSPNs in the plots reveals even more alarming discrepancies. Take, for example, He 2-131. Its terminal velocity would indicate a mass of about $0.6 M_{\odot}$ (circle 1A in Figure 3). But this mass is completely irreconcilable with its mass loss rate: it is found not at the position labelled $1 \mathrm{~A}$ in Figure 4 , but at $1 \mathrm{~B}$, with $\dot{M}$ a factor of hundred higher, suggesting a mass of above $0.94 M_{\odot}$ ! The reverse is true for NGC 2392. Its terminal velocity points to a mass of about $0.9 M_{\odot}$ (circle $2 \mathrm{~A}$ in Figure 3 ), but its observed mass loss rate is much too small for this mass (circle $2 \mathrm{~B}$ in Figure 4), indicating a mass of approximately $0.6 M_{\odot}$.

We still face a problem if we take the mass loss rate determinations of Kudritzki et al. to be correct: then our calculations would place these two stars at the positions labelled $1 \mathrm{~B}$ and $2 \mathrm{~B}$ in Figure 3 - with terminal velocities differing 
by a factor of 2 to 3 . But this is clearly ruled out by the observations. ( $v_{\infty}$ is a directly measurable quantity.)

We are therefore left with the conclusion that the analysis of Kudritzki et al. is at odds with the mass loss rates computed by our models based on the postAGB evolutionary tracks. To determine whether the reason for this discrepancy lies with the evolutionary tracks on the one hand or our hydrodynamical models and the analysis by Kudritzki et al. on the other requires further observational evidence. This is given to us by the UV spectra of the stars.

\section{Examples: UV analysis of the CSPNs He 2-131 and NGC 2392}

Figure 5 (top left) shows the synthetic UV spectrum of the model corresponding to $1 \mathrm{~A}$ in Figures 3 and 4 . It is clearly incompatible with the observed spectrum of He 2-131 (middle), since its mass loss rate is obviously too small, as evidenced by the presence of almost only purely photospheric lines hardly influenced by the thin wind - indicating that this CSPN must have a much larger luminosity, because $L$ is the major factor determining the mass loss rate. Thus, we have calculated a series of models with increasing luminosity - and therefore increasing mass loss rate - (at the same time adjusting the mass to keep the terminal velocity at its observed value) to see whether one of these models could reproduce the numerous strongly wind-contaminated iron lines observed especially in between 1500 and $1700 \AA$. Indeed, a model which due to its luminosity yields approximately the high mass loss rate of $1 \mathrm{~B}$ gives a much improved fit - see Figure 5, bottom left. (The parameters of this star are given in Table 1.)

The situation is reversed with NGC 2392. The synthetic spectrum of model $2 \mathrm{~A}$ is incompatible with the observed UV spectrum (Figure 5, right top and middle), since it produces many strongly wind-contaminated lines, which are, however, not observed. Instead, almost only photospheric lines are produced by the star. Again the problem is the luminosity, which in this case is much too high. Decreasing the luminosity and thus the mass loss rate yields a model with a much better agreement with the observed spectrum (Figure 5, bottom right).

What does this mean for the derived stellar parameters, which by virtue of this analysis now have the status of observed quantities? Let us consider first the weak-winded CSPN, NGC 2392. We determine a $T_{\text {eff }}$ of $40000 \mathrm{~K}$ from the ionization equilibrium of $\mathrm{Fe}$ ions in the stellar UV spectrum, not too different from the value obtained by the ionization equilibrium of $\mathrm{He}$ I and $\mathrm{He}$ II (absorption lines in the optical stellar spectrum). The very low terminal velocity in the wind of $400 \mathrm{~km} \mathrm{~s}^{-1}$ leads, together with a decreased luminosity (in order to reduce the predicted mass loss rate until the predicted and observed spectra are in good agreement), to a small radius. From this radius $\left(1.5 R_{\odot}\right)$ and $v_{\infty}$ we get a stellar mass of only $0.41 M_{\odot}$, a value much smaller than if we believe in the classical mass-luminosity relation - a high mass of $0.9 M_{\odot}$ was the result found by Kudritzki et al. (But due to the smaller $R$ and $T_{\text {eff }}$ our luminosity is also smaller.) We remark at this point that our error in the mass is extremely small $\left(\leq 0.1 M_{\odot}\right)$ due to the sensitive dependence on $v_{\infty}$ and the small error in this value $(\leq 10 \%)$. Furthermore, our predicted values of $v_{\infty}$ are in agreement within $10 \%$ with the observed values for the case of massive $\mathrm{O}$ stars (cf. Hoffmann and Pauldrach, these proceedings). Thus the systematic error is almost negligible. 

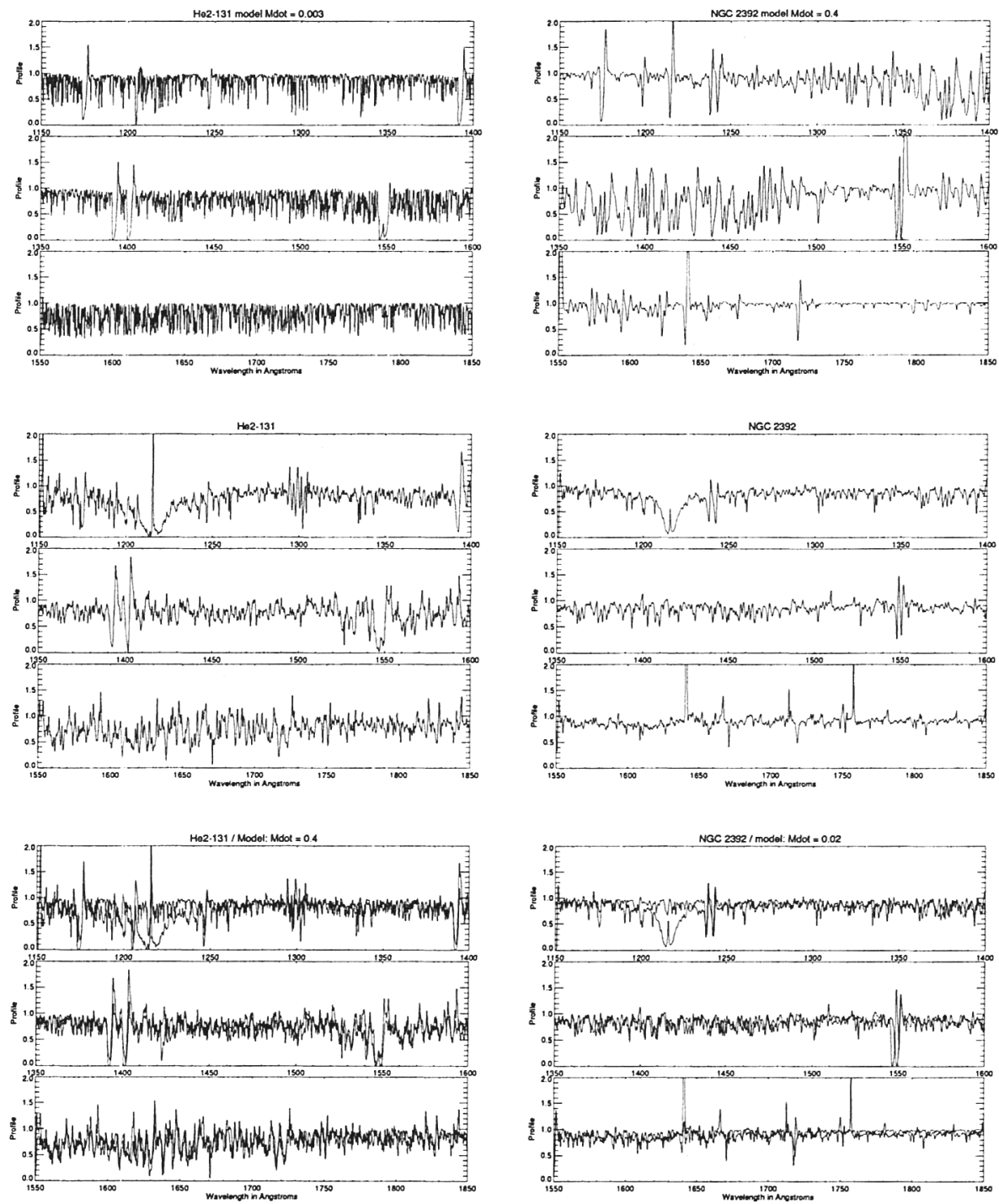

Figure 5. (Left) Top: Synthetic spectrum of model 1A for He 2-131 (see text). This is incompatible with the observed IUE spectrum (middle). A model with a significantly enhanced luminosity and thus mass loss rate reproduces the distinctive features in the UV spectrum much better (bottom, overplotted with the observed spectrum to better show the similarity). (Right) Top: Synthetic spectrum of model 2A for NGC 2392. Again, this is incompatible with the observed IUE spectrum (middle). In this case, however, the mass loss rate (and thus $L$ ) is much too high; a model with lower luminosity reproduces the spectrum much better (bottom). 
Table 1. Parameters of eight CSPNs derived by our analysis of the UV spectra using our model atmospheres, compared to the values found by Kudritzki et al. 1997.

\begin{tabular}{lcccccr}
\multicolumn{1}{c}{ Object } & $\begin{array}{c}T_{\text {eff }} \\
(\mathrm{K})\end{array}$ & $\begin{array}{c}R \\
\left(R_{\odot}\right)\end{array}$ & $\log \frac{L}{L_{\odot}}$ & $\begin{array}{c}M \\
\left(M_{\odot}\right)\end{array}$ & $\begin{array}{c}\dot{M} \\
\left(10^{-6} M_{\odot} / \mathrm{yr}\right)\end{array}$ & $\begin{array}{c}v_{\infty} \\
(\mathrm{km} / \mathrm{s})\end{array}$ \\
\hline NGC 2392 & 40000 & 1.5 & 3.7 & 0.41 & 0.018 & 420 \\
NGC 3242 & 75000 & 0.3 & 3.5 & 0.53 & 0.004 & 2400 \\
IC 4637 & 55000 & 0.8 & 3.7 & 0.87 & 0.019 & 1500 \\
IC 4593 & 40000 & 2.2 & 4.0 & 1.11 & 0.062 & 850 \\
He 2-108 & 39000 & 2.7 & 4.2 & 1.33 & 0.072 & 800 \\
Tc 1 & 35000 & 3.0 & 4.1 & 1.37 & 0.021 & 900 \\
He 2-131 & 33000 & 5.5 & 4.5 & 1.39 & 0.35 & 450 \\
NGC 6826 & 44000 & 2.2 & 4.2 & 1.40 & 0.18 & 1200 \\
\hline & \multicolumn{7}{c}{ Kudritzki et al. 1997 } \\
\hline NGC 2392 & 45000 & 2.5 & 4.4 & 0.91 & $\leq 0.03$ & 400 \\
NGC 3242 & 75000 & 0.6 & 4.0 & 0.66 & $\leq 0.02$ & 2300 \\
IC 4637 & 55000 & 1.3 & 4.1 & 0.78 & $\leq 0.02$ & 1500 \\
IC 4593 & 40000 & 2.2 & 4.0 & 0.70 & 0.1 & 900 \\
He 2-108 & 35000 & 3.2 & 4.1 & 0.75 & 0.24 & 700 \\
Tc 1 & 33000 & 5.1 & 4.4 & 0.95 & $\leq 0.1$ & 900 \\
He 2-131 & 30000 & 5.5 & 4.3 & 0.88 & 0.9 & 500 \\
NGC 6826 & 50000 & 2.0 & 4.3 & 0.92 & 0.26 & 1200 \\
\hline
\end{tabular}

Next we consider the central star of He 2-131. In this case the terminal velocity of $500 \mathrm{~km} \mathrm{~s}^{-1}\left(T_{\text {eff }}=33000 \mathrm{~K}\right)$ would appear to suggest, according to the classical post-AGB mass-luminosity relation, a stellar mass of about $0.6 M_{\odot}$ (cf. Figure 3). However, the wind features observed in the UV spectrum forced us to increase the stellar $R$ and $L$, which in turn increased $\dot{M}$ until a good fit was obtained. From the corresponding large radius $-5.5 R_{\odot}-$ and $v_{\infty}$ we derive a stellar mass of $1.39 M_{\odot}$, a value very close to the Chandrasekhar mass of relevance for type Ia supernovae. Thus, in this case the resulting mass is even more extreme than the value of $0.9 M_{\odot}$ obtained by Kudritzki et al.

\section{Interpretation of CSPN winds: results and discussion}

Table 1 shows the result of applying the method and UV analysis described in the previous section to eight CSPNs, including the two exemplary objects above. The problem which Table 1 points to is obvious: according to the postAGB evolutionary timescales we should not find so many extremely luminous CSPNs, because according to this theory they are expected to fade very quickly. We shall note, however, that the sample of objects chosen here is most likely not a representative one.

Figure 6 shows the relation between stellar mass and luminosity obtained from our model atmosphere analyses, in comparison with the mass-luminosity relation of the evolutionary tracks, represented by the values from Kudritzki et al. 1997. This plot shows that the problem already indicated is indeed very 


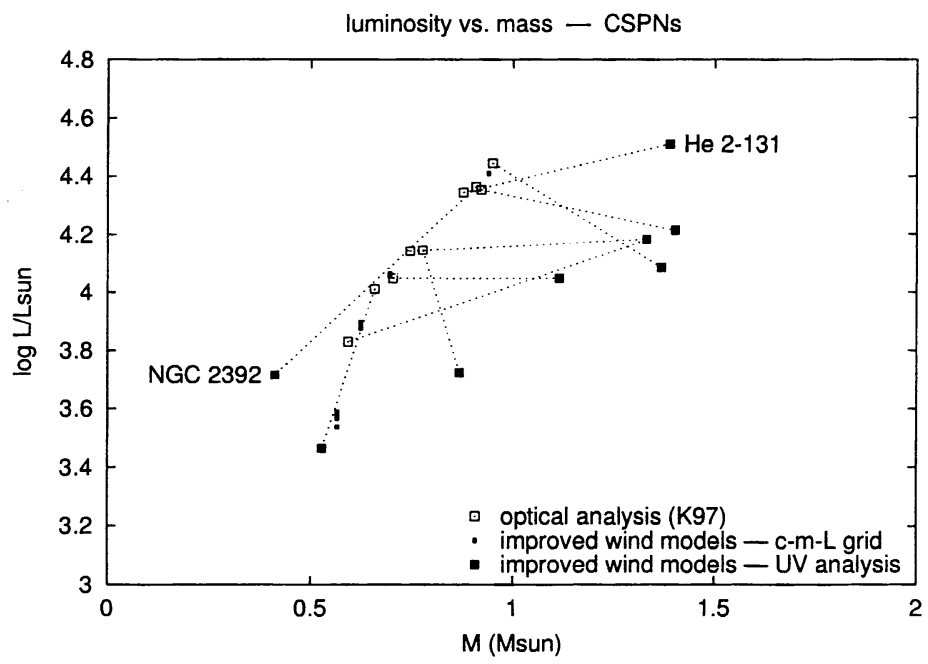

Figure 6. Luminosity vs. mass for the evolutionary tracks (open squares) compared to the observed quantities determined with our method (filled squares). Although the luminosities deduced from the UV spectra lie in the expected range, a much larger spread in the masses (from 0.4 to $1.4 M_{\odot}$ ) is obtained. No well-defined relation between CSPN mass and luminosity can be made out.

disturbing: the derived masses and luminosities do not agree with the classical post-AGB mass-luminosity relation. There is a very large spread in masses, between 0.4 and $1.4 M_{\odot}$, and there is no well-defined relation between CSPN mass and luminosity.

In Figure 7 we now again show the wind-momentum-luminosity relation for both massive hot stars and CSPNs, but this time based on the parameters derived in our analysis. Our models give wind momenta of the right order of magnitude and within the expected luminosity range (there may be too many CSPNs at $\log L / L_{\odot}>4$, but not so many as in Kudritzki et al. 1997). The CSPNs are found along the extrapolation of the wind-momentum-luminosity relation defined by the massive hot stars, and the CSPNs show a smaller dispersion, i. e., a tighter correlation of wind-momentum with luminosity, than was the case in Kudritzki et al. (1997). And, most important, this was achieved by fitting the multitude of diagnostic features in the CSPN UV spectra by means of up-to-date hydrodynamically consistent models.

How then does our wind-momentum-luminosity relation compare to that found by Kudritzki et al.? The answer is, quite favorably. If we drop the assumption made by Kudritzki et al. that the stars obey the theoretical postAGB mass-luminosity relation, and instead scale their mass loss rates to our radii $^{4}$ - keeping $Q$, the real observational quantity, fixed - then their wind

\footnotetext{
${ }^{4}$ Additionally allowing for their different effective temperatures by requiring that the observed visual flux $\left(\sim R^{2} T_{\text {eff }}\right)$ stay constant.
} 


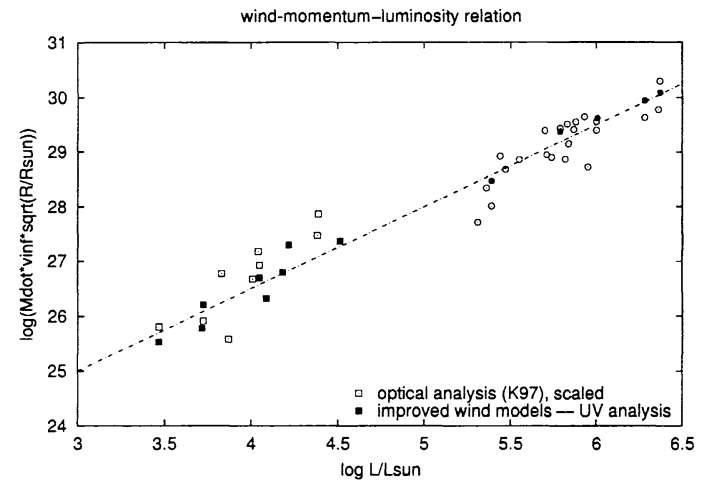

Figure 7. The wind-momentum-luminosity relation for CSPNs (lower left) based on our values determined from the UV spectra (filled squares). The open squares are the values from Kudritzki et al. with our radii applied (see text). Compared to Figure 2 the result is striking.

momenta match ours to within about a factor of two. Furthermore, their sample with the radii thus scaled now also shows a much tighter correlation of the wind momentum to luminosity than before (see Figure 7).

All this is strong evidence that all these winds are radiatively driven. It would be extremely difficult to explain the wind-momentum-luminosity relation if there was another mechanism driving the winds.

What makes the CSPN mass discrepancy problem found most intriguing is the fact that the same model atmospheres, with exactly the same physics, obviously work also perfectly well for massive $\mathrm{O}$ stars, as we have shown. We are reluctant to conclude that there is something basic we do not understand about either the winds and photospheres of O-type stars, or how to produce CSPNs and what their internal structure is. Both alternatives are difficult to believe. But we need to explain why we obtain such a spread in the masses.

We cannot offer a fair solution to this paradox; all we can do right now is to present our method, the results obtained from our analysis, and the corresponding problem in the clearest possible way, which is usually the first step along the road that leads to the solution.

\section{References}

Blöcker T., A\&A 299, 755 (1995)

Hoffmann T. L., Pauldrach A.W. A., these proceedings

Kudritzki R.-P., Méndez R. H., Puls J., McCarthy, J. K., in IAU Symp. 180, Planetary Nebulae, eds. H. J. Habing \& H. J. G. L. M. Lamers, p. 64 (1997)

Méndez R. H., Kudritzki R.-P., Herrero A., A\&A 260, 329 (1992)

Pauldrach A. W. A., Puls J., Kudritzki R.-P., et al., A\&A 207, 123 (1988)

Pauldrach A. W. A., Hoffmann T.L., Lennon, M., A\&A 375, 161 (2001)

Pauldrach A. W. A., Hoffmann T. L., A\&A in press (2002)

Puls J., Kudritzki R.-P., Herrero A., et al., A\&A 305, 171 (1996)

Scuderi S., Panagia N., Stanghellini C., et al., A\&A 332, 251 (1998) 